\title{
Biosynthesis of heparin
}

\section{Modulation of polysaccharide chain length in a cell-free system}

\author{
Kerstin LIDHOLT, ${ }^{*} \ddagger$ Johan RIESENFELD, ${ }^{*}$ Karl-Gustav JACOBSSON, ${ }^{*}$ David S. FEINGOLD $\dagger$ \\ and UIf LINDAHL* \\ *Department of Veterinary Medical Chemistry, The Swedish University of Agricultural Sciences, The Biomedical Center, \\ Box 575, S-751 23 Uppsala, Sweden, and †Department of Microbiology, School of Medicine, University of Pittsburgh, \\ Pittsburgh, PA 15261, U.S.A.
}

\begin{abstract}
The formation of heparin-precursor polysaccharide ( $N$-acetylheparosan) was studied with a mouse mastocytoma microsomal fraction. Incubation of this fraction with UDP- $\left[{ }^{3} \mathrm{H}\right] \mathrm{GlcA}$ and UDP-GlcNAc yielded labelled macromolecules that could be depolymerized, apparently to single polysaccharide chains, by alkali treatment, and thus were assumed to be proteoglycans. Label from UDP- $\left[{ }^{3} \mathrm{H}\right] \mathrm{GlcA}$ (approx. $3 \mu \mathrm{M}$ ) is transiently incorporated into microsomal polysaccharide even in the absence of added UDP-GlcNAc, probably owing to the presence of endogenous sugar nucleotide. When the concentration of exogenous UDP-GlcNAc was increased to $25 \mu \mathrm{M}$ the rate of incorporation of ${ }^{3} \mathrm{H}$ increased and proteoglycans carrying polysaccharide chains with an $M_{\mathrm{r}}$ of approx. 110000 were produced. Increasing the UDP-GlcNAc concentration to $5 \mathrm{~mm}$ led to an approx. 4-fold decrease in the rate of ${ }^{3} \mathrm{H}$ incorporation and a decrease in the $M_{\mathrm{r}}$ of the resulting polysaccharide chains to approx. 6000 (predominant component). When both UDPGlcA and UDP-GlcNAc were present at high concentrations $(5 \mathrm{~mm})$ the rate of polymerization and the polysaccharide chain size were again increased. The results suggest that the inhibition of polymerization observed at grossly different concentrations of the two sugar nucleotides, UDP-GlcA and UDP-GlcNAc, may be due either to interference with the transport of one of these precursors across the Golgi membrane or to competitive inhibition of one of the glycosyltransferases. The maximal rate of chain elongation obtained, under the conditions employed, was about 40 disaccharide units $/ \mathrm{min}$. The final length of the polysaccharide chains was directly related to the rate of the polymerization reaction.
\end{abstract}

\section{INTRODUCTION}

The sulphated glycosaminoglycans generally occur in the tissues as proteoglycans composed of polysaccharide chains covalently linked to a protein core (Rodén, 1980; Gallagher et al., 1986; Poole, 1986). For all proteoglycan species, except keratan sulphate, the linkage region consists of a galactosyl-galactosyl-xylosyl trisaccharide sequence, which connects the polysaccharide chain to a serine residue in the core protein. Analysis of single polysaccharide chains released from proteoglycans by alkaline $\beta$-elimination has revealed striking variation in chain length, not only between proteoglycans of different origin or polysaccharide structure, but also within single proteoglycan preparations. The polysaccharide chain length influences the physicochemical properties of the proteoglycan and thus is a parameter of potential functional importance.

The biosynthesis of proteoglycans is believed to be initiated by formation of the core polypeptide, which then becomes glycosylated (Rodén, 1980). After formation of the trisaccharide linkage region the actual polysaccharide is assembled by alternate transfer of Dglucuronosyl and $N$-acetyl-D-hexosaminyl moieties from the appropriate UDP-sugars to the non-reducing end of the growing chain. Following these glycosylation reactions, the polysaccharide chains undergo modification, which includes the incorporation of sulphate groups and yields the mature proteoglycan. During the polymer- ization and polymer-modification reactions both the proteoglycan intermediates and the enzymes involved appear to be bound to the endoplasmic, presumably Golgi, membranes. Although some basic features of these reactions have been defined (Rodén, 1980; Lindahl \& Kjellén, 1986), major aspects of regulation remain unclear. This applies also to the mechanisms that control the polymerization reactions and determine the length of the resulting polysaccharide chains.

The present paper is concerned with chain-elongation reactions in a microsomal fraction from a heparinsynthesizing mouse mastocytoma. It is shown that the rate of polymerization can be modulated extensively by varying the concentrations of the UDP-sugar substrates added to the system. Furthermore, this modulation is strikingly reflected in the length of the synthesized polysaccharide chains.

\section{EXPERIMENTAL}

\section{Materials}

A microsomal fraction was prepared as described previously (Jacobsson et al., 1979a) from a transplantable mouse mastocytoma (Furth et al., 1957). UDP- $\left.{ }^{3} \mathrm{H}\right] \mathrm{GlcA}$ was synthesized enzymically from $D-\left[1-{ }^{3} \mathrm{H}\right]$ glucose $(15 \mathrm{Ci} / \mathrm{mmol}$; New England Nuclear, Boston, MA, U.S.A.) as reported previously (Jacobsson et al., 1979a). Unlabelled UDP-GlcA, UDP-GlcNAc, other nucleo-

$\ddagger$ To whom correspondence should be addressed. 
tides, chondroitin $\mathrm{ABC}$ lyase (chondroitinase $\mathrm{ABC}$ ) and bovine liver $\beta$-D-glucuronidase (type B-10) were obtained from Sigma Chemical Co. (St. Louis, MO, U.S.A.). Hyaluronan [see Balazs et al. (1986) for nomenclature] fractions ranging in average $M_{\mathrm{r}}$ from approx. 6600 to approx. 43000 , isolated from a partially hydrolysed sample (Cleland, 1983), were provided by R. Cleland (Dartmouth College, Hanover, NH, U.S.A.). Two fractions, $M_{\mathrm{r}}$ approx. 170000 and approx. 210000, obtained by autoclaving hyaluronan at $128^{\circ} \mathrm{C}$, were given by $\mathrm{O}$. Wik (Pharmacia Fine Chemicals, Uppsala, Sweden).

\section{Methods}

The formation of heparin-precursor polysaccharide was studied by incubating $50 \mu \mathrm{Ci}$ (approx. $3 \mathrm{nmol}$ ) of UDP- $\left[{ }^{3} \mathrm{H}\right] \mathrm{GlcA}$ and various amounts of UDP-GlcNAc with approx. $10 \mathrm{mg}$ of microsomal protein per $\mathrm{ml}$ of

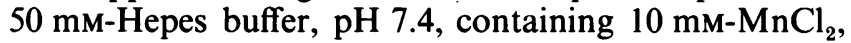
$10 \mathrm{~mm}-\mathrm{MgCl}_{2}$ and $5 \mathrm{~mm}-\mathrm{CaCl}_{2}$. After incubation at $37^{\circ} \mathrm{C}$ for various periods of time the reactions were interrupted by the addition of SDS to a final concentration of $2 \%(\mathrm{w} / \mathrm{v})$, and were then heated at $100^{\circ} \mathrm{C}$ for $3 \mathrm{~min}$. Labelled polysaccharide was isolated along with $0.5 \mathrm{mg}$ of carrier heparin from portions $(100-300 \mu \mathrm{l})$ of incubation mixtures by passage through columns $(1 \mathrm{~cm} \times 35 \mathrm{~cm})$ of Sephadex G-50 (medium grade) equilibrated with $50 \mathrm{~mm}$-Tris/ $\mathrm{HCl}$ buffer, $\mathrm{pH} \mathrm{8.0,} \mathrm{containing}$ $0.1 \mathrm{M}-\mathrm{NaCl}$ and $0.1 \%$ SDS. Fractions $(2 \mathrm{ml})$ were collected at a rate of $12 \mathrm{ml} / \mathrm{h}$ and analysed for radioactivity by liquid-scintillation counting; labelled proteoglycan appeared as a distinct peak at the excluded volume of the column.

To convert proteoglycan into single polysaccharide chains $\mathrm{NaOH}$ was added to a final concentration of $0.5 \mathrm{M}$ and the sample was kept at room temperature for $20 \mathrm{~h}$. Control experiments using gel chromatography showed that the released labelled polysaccharide chains were not depolymerized to any significant extent during alkali treatment. After completed $\beta$-elimination the samples were passed through Sep-Pak $C_{18}$ cartridges (Waters Associates, Milford, MA, U.S.A.) in order to eliminate the SDS. The samples were concentrated as required for further analysis by using a Speed Vac evaporation centrifuge (Savant Instruments, Hickville, NY, U.S.A.). An alternative method for preparing single polysaccharide chains, involving digestion of the microsomal incubation mixtures with Pronase followed by alkali treatment, was abandoned because of consistently lower recoveries of the largest molecules.

Gel chromatography was performed on a column $(1 \mathrm{~cm} \times 80 \mathrm{~cm})$ of Sepharose 2B-CL equilibrated with $1 \mathrm{M}$ $\mathrm{NaCl}$. Fractions (approx. $1.7 \mathrm{ml}$ ) were collected at a rate of $5 \mathrm{ml} / \mathrm{h}$. Free polysaccharide chains were also analysed on columns $(0.75 \mathrm{~cm} \times 60 \mathrm{~cm})$ of Ultropac TSK G4000 SW and TSK G2000 SW (LKB, Bromma, Sweden) equilibrated with $1 \mathrm{M}-\mathrm{NaCl}$ in $10 \mathrm{~mm}-\mathrm{Tris} / \mathrm{HCl}$ buffer, $\mathrm{pH}$ 7.4. The columns were eluted at a flow rate of $0.5 \mathrm{ml} /$ min by means of an LKB model 2150 h.p.l.c. pump, and $1 \mathrm{ml}$ fractions were collected. $V_{0}$ and $V_{\mathrm{t}}$ were determined by using hyaluronan (Healon; Pharmacia) and ${ }^{3} \mathrm{H}_{2} \mathrm{O}$ respectively. The columns were calibrated with hyaluronan fractions of defined $M_{\mathrm{r}}$ (see the Experimental section), which were detected by the carbazole reaction (Bitter \& Muir, 1962). Differences in polysaccharide chain size revealed by this system (see the Results section) were maintained also when the eluent was changed to $4 \mathrm{M}$-guanidinium chloride (results not shown).

\section{RESULTS}

Previous studies with mastocytoma microsomal fractions have demonstrated synthesis of a heparin-precursor polysaccharide from the appropriate nucleotide sugars (Silbert 1963; Lindahl et al., 1973; Höök et al., 1975; Jacobsson \& Lindahl, 1980). Accordingly, the labelled macromolecular product (excluded from Sephadex G50) formed by incubating a microsomal preparation with UDP- $\left[{ }^{3} \mathrm{H}\right] \mathrm{GlcA}$ and UDP-GlcNAc was largely (invariably $>85 \%$ ) resistant to degradation by chondroitin lyase $\mathrm{ABC}$, but susceptible to deaminative cleavage by $\mathrm{HNO}_{2}$ at pH 3.9 (results not shown). In previous experiments the incorporation of radioactivity from labelled UDP-GlcA into microsomal polysaccharide remained essentially linear with time for more than $1 \mathrm{~h}$, provided that the concentration of UDPGlcNAc was sufficiently high ( $>2.5 \mathrm{~mm}$; Lindahl et al., 1973). This system has now been used to study in more detail the kinetics of the polymerization reaction.

\section{Incorporation of $\left[{ }^{3} \mathbf{H}\right]$ glucuronic acid into microsomal polysaccharide}

Incubation of the mastocytoma microsomal fraction with UDP- $\left[{ }^{3} \mathrm{H}\right] \mathrm{GlcA}$ in the presence of $5 \mathrm{~mm}$ unlabelled UDP-GlcNAc yielded labelled polysaccharide at a constant rate for at least $1 \mathrm{~h}$ (Fig. 1; cf. Lindahl et al., 1973). When the concentration of UDP-GlcNAc was decreased to $25 \mu \mathrm{M}$ the incorporation of label into polysaccharide started to level off after about $10 \mathrm{~min}$ and ceased altogether after $30-40 \mathrm{~min}$ (Fig. 1). However,

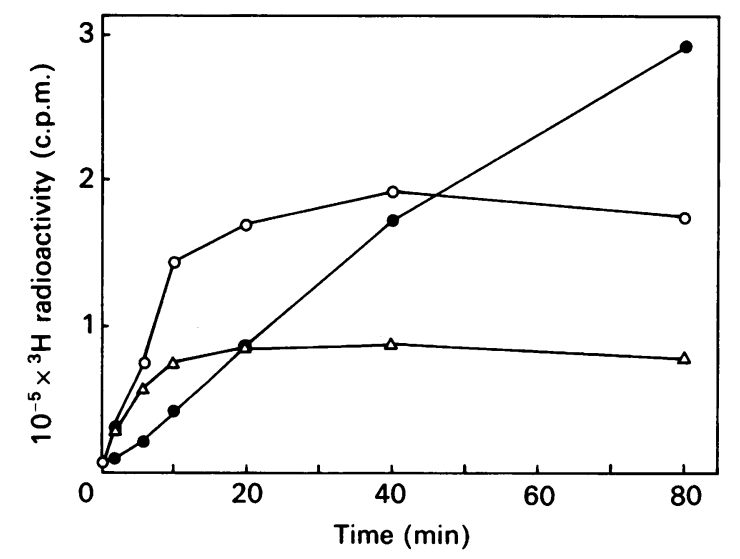

Fig. 1. Time course of incorporation of radioactivity from UDP$\left.\right|^{3} \mathrm{H} \mid$ GlcA into microsomal polysaccharide at different UDP-GlcNAc concentrations

Mastocytoma microsomal fractions was incubated at $37^{\circ} \mathrm{C}$ with UDP- $\left[{ }^{3} \mathrm{H}\right] \mathrm{GlcA}$ and $25 \mu \mathrm{M}-(\mathrm{O}), 5 \mathrm{~mm}-(\mathrm{O})$ or no $(\triangle)$ exogenous UDP-GlcNAc as described in the Experimental section. After the indicated periods samples of $300 \mu \mathrm{l}$ were withdrawn and labelled polysaccharide was isolated by gel chromatography (see the Experimental section). The values are corrected for non-specific incorporation of radioactivity (approx. 25000 c.p.m. of ${ }^{3} \mathrm{H} /$ sample), which was quantified by using a control incubation with heat-inactivated enzyme. For further information see the text. 
during the period of linear incorporation, i.e. the first 10 min of incubation, the rate of polysaccharide synthesis was 4.3-fold higher (average of 12 experiments; S.D. \pm 1.3 ) in the presence of $25 \mu \mathrm{M}$-UDP-GlcNAc than in the presence of $5 \mathrm{~mm}$-UDP-GlcNAc. Significant polymerization was observed even in the absence of added UDP-GlcNAc, suggesting the presence of endogenous UDP-GlcNAc in the microsomal preparation (Fig. 1). Again the rate of polysaccharide formation was initially higher than that observed with $5 \mathrm{~mm}$-UDP-GlcNAc but levelled off after about $10 \mathrm{~min}$ of incubation, the final amount of polysaccharide synthesized being about half of that produced in the presence of $25 \mu \mathrm{M}$ exogenous UDP-GlcNAc. The decrease in incorporation rate noted at low UDP-GlcNAc concentration, following the initial $10 \mathrm{~min}$ incubation period, coincided with depletion of the UDP- $\left[{ }^{3} \mathrm{H}\right] \mathrm{GlcA}$ pool, as indicated by high-voltage paper electrophoresis of samples of the incubation mixtures (results not shown). During incubation the UDP- $\left[{ }^{3} \mathrm{H}\right] \mathrm{GlcA}$ was gradually replaced by a fastermigrating labelled component, presumably $\left[{ }^{3} \mathrm{H}\right] \mathrm{GlcA}$ 1-phosphate. Apparently, the labelled UDP-GlcA was protected from enyzmic degradation at higher concentrations of UDP-GlcNAc.

The evaluation of these results was complicated by the presence of labelled material that appeared to be formed through a non-enzymic mechanism. This material was recovered from incubations containing microsomal protein that had been heated in $2 \%$ SDS at $100^{\circ} \mathrm{C}$ for $10 \mathrm{~min}$ before the addition of sugar nucleotides. It was excluded from Sephadex G-50 in the initial isolation step, but appeared as a small molecular component, diffusible on dialysis and well-retarded on renewed Sephadex G-50 chromatography after alkali treatment of the proteoglycan fraction. The amounts of this component formed were remarkably constant, approx. 25000 c.p.m. in a $300 \mu \mathrm{l}$ standard incubation, and were related to the amounts of microsomal protein present. This apparent artifact, which has not been identified, is indicated in gel chromatograms of free polysaccharide chains as retarded shaded peaks (see Figs. 5 and 6), and has been appropriately corrected for in the kinetic analyses (Figs. 1 and 2 and Table 1).

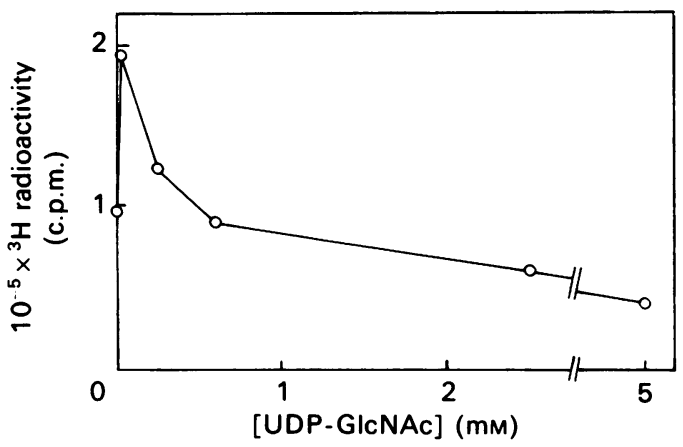

Fig. 2. Formation of microsomal polysaccharide as a function of UDP-GlcNAc concentration

Mastocytoma microsomal fraction was incubated in a total volume of $300 \mu \mathrm{l}$ with UDP- $\left[{ }^{3} \mathrm{H}\right] \mathrm{GlcA}$ and UDPGlcNAc at the concentrations indicated, as described in the Experimental section. After $5 \mathrm{~min}$ at $37^{\circ} \mathrm{C}$ the incubations were interrupted and labelled polysaccharide was isolated. Values are corrected for non-specific incorporation of radioactivity (see the legend to Fig. 1).
Table 1. Effects of nucleotides on polysaccharide formation

Standard incubation mixtures (see the Experimental section) of $200 \mu \mathrm{l}$, containing UDP- $\left[{ }^{3} \mathrm{H}\right] \mathrm{GlcA}$ and $25 \mu \mathrm{M}-$ UDP-GlcNAc (control), as well as similar mixtures with the additions indicated, were kept at $37^{\circ} \mathrm{C}$ for $10 \mathrm{~min}$ and labelled polysaccharide was isolated by gel chromatography on Sephadex G-50. The control incubation mixture incorporated 290000 c.p.m. of ${ }^{3} \mathrm{H}$ from UDP- $\left[{ }^{3} \mathrm{H}\right] \mathrm{GlcA}$ into microsomal polysaccharide. The values indicated are averages of two separate experiments and are corrected with regard to non-enzymic incorporation of radioactivity into macromolecular material (see the legend to Fig. 1).

Addition
$\left[{ }^{3} \mathrm{H}\right]$ Polysaccharide formed
$(\%$ of control $)$

\begin{tabular}{lr}
\hline & 100 \\
None (control) & 29 \\
5 mM-UDP-GlcNAc & 10 \\
5 mM-UTP & 12 \\
5 mM-UDP & 25 \\
5 mM-UMP & 100 \\
5 mM-Uridine & 62 \\
5 mM-ATP & 48 \\
5 mM-ADP & 42 \\
5 mM-AMP & 101 \\
5 mM-Pyrophosphate &
\end{tabular}

The effect of UDP-GlcNAc concentration on the rate of polymerization was studied in more detail by determining the amounts of labelled polysaccharide formed in the presence of UDP-[ $\left.{ }^{3} \mathrm{H}\right] \mathrm{GlcA}$ (approx. $3 \mu \mathrm{M}$; see below) during the initial $5 \mathrm{~min}$ of incubation. The resulting activity profile showed a sharp highly reproducible maximum at about $25 \mu \mathrm{M}$-UDP-GlcNAc, with a consistent decline in rate with higher concentrations of this precursor (Fig. 2). Maximal rate of polymerization was maintained even with $2.5 \mu \mathrm{M}$-UDPGlcNAc (results not shown), whereas a lower rate was obtained in the absence of exogenous UDP-GlcNAc (significance uncertain because of apparent deviation from linear incorporation kinetics; see Fig. 1). In a particular experiment, the actual rate of polysaccharide formation at $25 \mu \mathrm{M}$-UDP-GlcNAc was calculated as approx. $0.6 \mathrm{pmol}$ of hexuronic acid incorporated (disaccharide unit formed)/min per $\mathrm{mg}$ of microsomal protein, assuming a specific radioactivity for the added UDP- $\left[{ }^{3} \mathrm{H}\right] \mathrm{GlcA}$ of $15 \mathrm{Ci} / \mathrm{mmol}$ and no endogenous UDP-GlcA, hence a UDP-GlcA concentration of approx. $3 \mu \mathrm{M}$. In the presence of $5 \mathrm{~mm}$-UDP-GlcNAc the corresponding rate declined to $\sim 0.15 \mathrm{pmol} / \mathrm{min}$ per $\mathrm{mg}$ of protein. When unlabelled UDP-GlcA was added to incubation mixtures containing UDP- $\left[{ }^{3} \mathrm{H}\right] \mathrm{GlcA}$ and $5 \mathrm{mM}$-UDP-GlcNAc, the amount of ${ }^{3} \mathrm{H}$ incorporated into polysaccharide decreased, owing to the decrease in specific radioactivity of the UDP- $\left[{ }^{3} \mathrm{H}\right]$ GlcA pool. However, the decrease in the rate of ${ }^{3} \mathrm{H}$ incorporation was much smaller than was expected from the dilution of the UDP- $\left[{ }^{3} \mathrm{H}\right]$ GlcA pool, suggesting a substantial increase in the overall rate of polysaccharide synthesis. An incubation mixture containing $5 \mathrm{~mm}$-UDP-GlcA along with $5 \mathrm{~mm}$-UDP-GlcNAc thus synthesized polysaccharide at an apparent rate of approx. $15 \mathrm{pmol} / \mathrm{min}$ per mg of protein. These findings suggest that the UDP$\left[{ }^{3} \mathrm{H}\right] \mathrm{Glc} A$ concentration utilized in most of the experiments fell well below the saturation value for the glucuronosyltransferase; in fact, a $K_{\mathrm{m}}$ of approx. $50 \mu \mathrm{M}$ was 
calculated for UDP-GlcA in a previous similar experiment at $2.5 \mathrm{~mm}$-UDP-GlcNAc concentration (see Lindahl et al., 1973). (It should be realized, however, that the reliability of such $K_{\mathrm{m}}$ determinations is limited in view of the particular nature of the biosynthetic system.)

Various nucleotides were tested with regard to their effects on polysaccharide synthesis in the microsomal

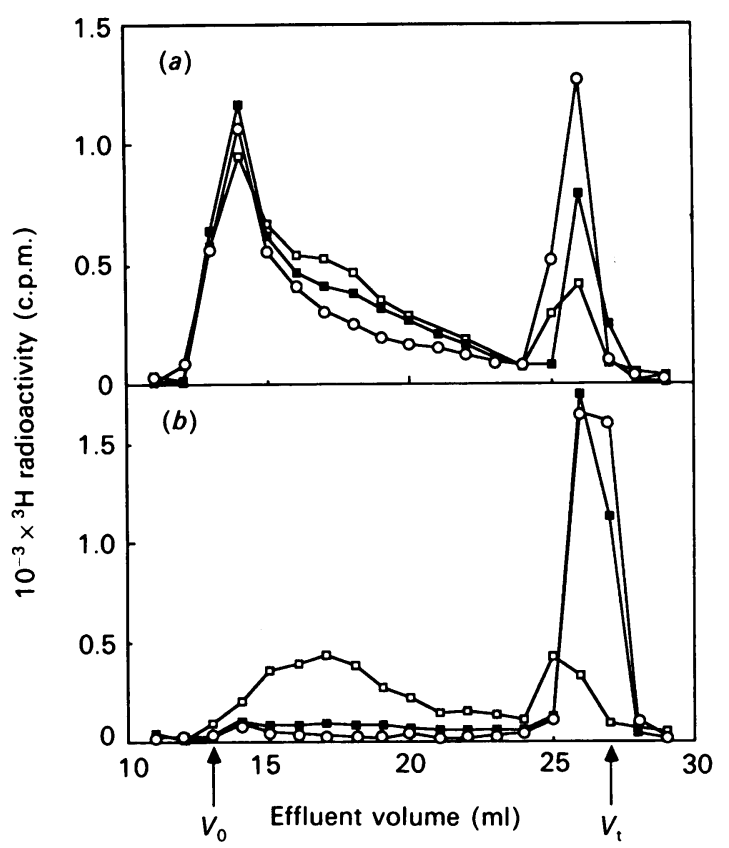

Fig. 3. Effect of Triton $\mathrm{X}-100$ on the formation of microsomal polysaccharide

Mastocytoma microsomal fraction was incubated (500 $\mu$ l incubation mixtures) for $30 \mathrm{~min}(a)$ with UDP- $\left[{ }^{3} \mathrm{H}\right] \mathrm{GlcA}$ and $5 \mathrm{~mm}$-UDP-GlcNAc under standard conditions (see the Experimental section) or (b) with UDP- $\left[{ }^{3} \mathrm{H}\right] \mathrm{GlcA}$ alone in the presence of $1 \%$ Triton $X-100$. Labelled macromolecules were isolated by gel chromatography and were then treated with alkali, as described in the Experimental section. The products were adsorbed on small columns $(1 \mathrm{~cm} \times 2 \mathrm{~cm})$ of DEAE-cellulose (Whatman DE-52), equilibrated with $50 \mathrm{~mm}-\mathrm{Tris} / \mathrm{HCl}$ buffer, $\mathrm{pH} 8.0$, which were then washed with $10 \mathrm{ml}$ of $50 \mathrm{~mm}-\mathrm{LiCl}$ in $50 \mathrm{~mm}$-acetate buffer, $\mathrm{pH}$ 4.0. This procedure eliminated most of the artifact product(s) due to non-enzymic incorporation of label (see the Results section). Remaining labelled material [approx. 300000 c.p.m. from experiment $(a)$ and 50000 c.p.m. from experiment $(b)]$ was eluted with

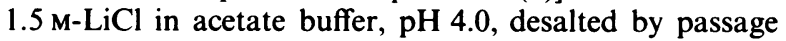
through Sephadex G-25 (PD-10; Pharmacia) columns, and analysed by gel chromatography on TSK G2000 SW as described in the Experimental section ( $\square$ ). Before chromatography, samples of labelled material were digested with $\beta$-glucuronidase (in accordance with Jacobsson et al., 1979b) ( $\square$ ) or with chondroitin ABC lyase (in accordance with Jacobsson \& Lindahl, 1987) (O). The retarded components appearing at approx. $25 \mathrm{ml}$ effluent volume have not been identified. They may represent a remainder of the artifactual labelled material (see the Results section), most of which was removed with the breakthrough and washing of the fractions off the DEAE-cellulose column; in fact, separate analysis of each of these fractions on the TSK 2000 column showed exclusively low- $M_{\mathrm{r}}$ components, with an elution position similar to those displayed in the Figure. system (Table 1). The nucleotides were added at $5 \mathrm{~mm}$ to standard incubation mixtures containing UDP- $\left[{ }^{3} \mathrm{H}\right] \mathrm{GlcA}$ and $25 \mu \mathrm{M}$-UDP-GlcNAc. Under these conditions not only UDP-GlcNAc (at $5 \mathrm{~mm}$ ), but also, and to a larger extent, the uridine mono-, di- and tri-phosphates lacking the sugar residue, lowered the rate of polysaccharide synthesis. Uridine and pyrophosphate had no effect. The adenosine-based nucleotides showed significant inhibitory effect, albeit less pronounced than that of uridine nucleotides.

Experiments were undertaken to ascertain that the incorporation of labelled glucuronic acid did indeed represent a true polymerization reaction, and not merely capping of preformed primer molecules with a labelled monosaccharide residue at the non-reducing end. Labelled polysaccharide isolated after incubation of microsomal fraction with UDP- $\left[{ }^{3} \mathrm{H}\right] \mathrm{GlcA}$ and $5 \mathrm{~mm}-$ UDP-GlcNAc for $30 \mathrm{~min}$ was essentially resistant to digestion with bovine liver $\beta$-glucuronidase (Fig. 3a), showing that most of the labelled glucuronic acid units had become incorporated into internal positions of the polysaccharide chain. The product obtained in the absence of exogenous UDP-GlcNAc released only a small portion, about $15 \%$, of the incorporated uronic acid (results not shown), in agreement with the notion that the microsomal preparation contained appreciable amounts of endogenous UDP- $N$-acetylhexosamine. For comparison, a corresponding incubation was performed in the presence of $1 \%$ Triton X-100, a non-ionic detergent previously shown to preclude elongation of the endogenous microsomal polysaccharide without interfering with the individual glycosyltransferase reactions (Riesenfeld et al., 1987). The resulting labelled product released free $\left[{ }^{3} \mathrm{H}\right]$ glucuronic acid monsaccharide in almost quantitative yield on digestion with $\beta$-glucuronidase (Fig. 3b), as predicted for a non-reducing terminal glucuronic acid residue. This product was of particular interest, since it represented endogenous glycosyl acceptors (' primers') serving as potential sites of polysaccharide chain elongation in the intact microsomal system, and was therefore analysed further. The endgroup-labelled species, $M_{\mathrm{r}}$ approx. 12000 , emerged as a sulphated polysaccharide on DEAE-cellulose ionexchange chromatography (results not shown), and was virtually all susceptible to degradation by chondroitin ABC lyase (Fig. $3 b$ ). These findings suggested that the major component capable of serving as a glucuronosyl acceptor in the solubilized system was a chondroitin sulphate. In contrast, most of the labelled polysaccharide produced in the intact microsomal system (in the presence of UDP-GlcNAc) resisted chondroitin lyase digestion (Fig. $3 a$ ), as expected for a heparin precursor. Lacking any demonstrable heparin-related glycosyl acceptor in the microsomal fraction, it is conceivable that such structures were formed de novo during the incubations, starting from preformed polysaccharide-protein linkage regions.

\section{Effects of UDP-sugar concentration on polysaccharide chain length}

The macromolecular properties of the microsomal polysaccharide were initially investigated by using gel chromatography on Sepharose 2B. The product labelled by UDP- $\left[{ }^{3} \mathrm{H}\right] \mathrm{GlcA}$ in the presence of $25 \mu \mathrm{M}$-UDPGlcNAc emerged considerably before that synthesized 


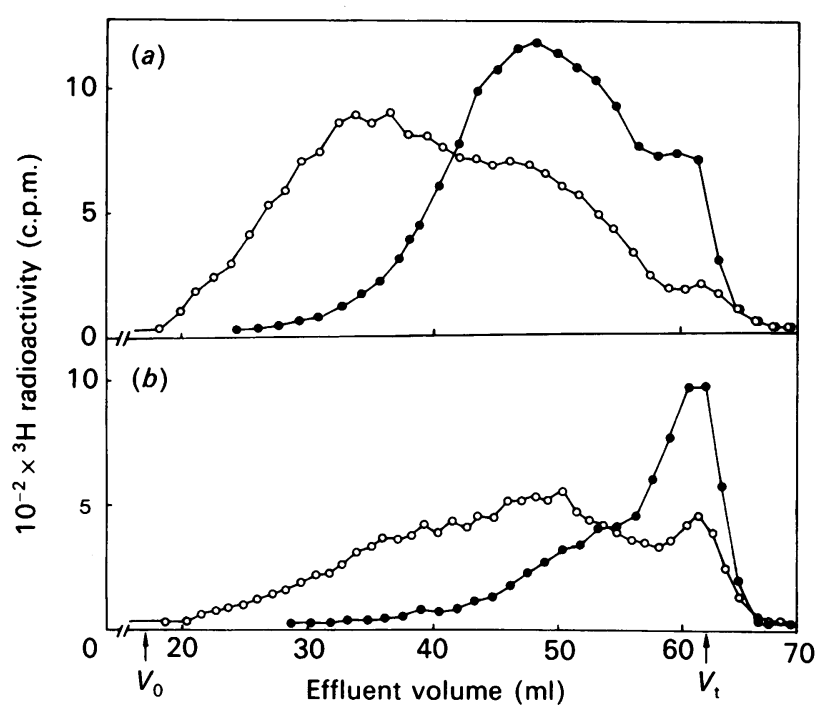

Fig. 4. Effect of alkali treatment on the macromolecular properties of microsomal polysaccharide

Microsomal fraction was incubated under standard conditions (see the Experimental section) in a total volume of $300 \mu \mathrm{l}$ with UDP- $\left[{ }^{3} \mathrm{H}\right] \mathrm{GlcA}$ and either $(a) 25 \mu \mathrm{M}$ - or $(b)$ $5 \mathrm{~mm}$-UDP-GlcNAc. After $5 \mathrm{~min}$ at $37^{\circ} \mathrm{C}$ the incubations were interrupted and labelled macromolecules were isolated by gel chromatography on Sephadex G-50 (see the Experimental section). Distinct fractions of excluded material (not shown) were recovered and were then analysed by gel chromatography on Sepharose 2B, either before (O) or after (O) alkali treatment (see the Experimental section). Each sample applied contained approx. 17000 c.p.m. of ${ }^{3} \mathrm{H}$. Part of the most retarded peaks of the alkali-treated samples $(O)$ represents nonspecific incorporation of label. For further information see the text.

in the presence of $5 \mathrm{~mm}$-UDP-GlcNAc (Fig. 4). The polysaccharide chains released from the macromolecules by alkali treatment appeared at more retarded elution positions, with the chains synthesized in the presence of $25 \mu \mathrm{M}$-UDP-GlcNAc again being appreciably larger than those obtained with 5 mM-UDP-GlcNAc (Fig. 4). These findings indicate, as expected (Robinson et al., 1978), but not previously demonstrated, that the heparinprecursor polysaccharide is synthesized as a proteoglycan in which the individual polysaccharide chains are bound by alkali-sensitive linkages. However, they also point to an unexpected correlation between the rate of the polymerization reaction and the molecular size of the resulting proteoglycan, since proteoglycan molecules synthesized at a high rate (in the presence of $25 \mu \mathrm{M}$-UDPGlcNAc) were larger than those synthesized at a lower rate (in the presence of $5 \mathrm{~mm}$-UDP-GlcNAc). The concomitant difference in elution position of the corresponding alkali-treated products indicates that the modulation of proteoglycan size is due, at least partly, to a change in length of the constituent polysaccharide chains.

A more detailed analysis of polysaccharide chain length revealed striking differences related to the concentration of UDP-GlcNAc during polymerization (Fig. 5). After $5 \mathrm{~min}$ of incubation in the presence of UDP$\left[{ }^{3} \mathrm{H}\right] \mathrm{GlcA}$ (approx. $3 \mu \mathrm{M}$ ) and low concentrations of UDPGlcNAc $(2.5 \mu \mathrm{M}$ or $25 \mu \mathrm{M}$ exogenous sugar nucleotide)

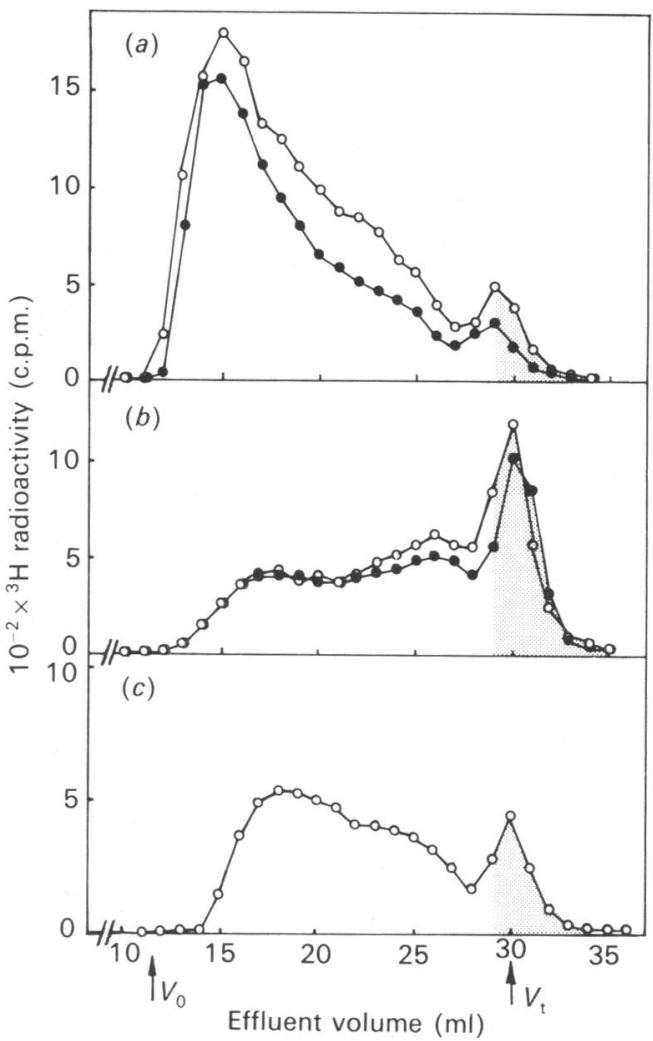

Fig. 5. Gel chromatography of polysaccharide chains synthesized at different UDP-GlcNAc concentrations

Standard incubation mixtures of $300 \mu$ containing UDP$\left[{ }^{3} \mathrm{H}\right] \mathrm{GlcA}$ and $(a) 2.5 \mu \mathrm{M}-(\bigcirc)$ or $25 \mu \mathrm{M}-(\bigcirc),(b) 2.5 \mathrm{~mm}-$ (O) or $5 \mathrm{~mm}-(O)$, or (c) no exogenous UDP-GlcNAc, were kept at $37^{\circ} \mathrm{C}$ for $5 \mathrm{~min}$ and labelled microsomal polysaccharide was isolated. After alkali treatment the resulting free polysaccharide chains were subjected to chromatography on a column of Ultropac TSK G4000 SW. The stippled peaks represent at least partly nonspecific incorporation of label that was recovered with the macromolecular fraction and released as (a) smallmolecular component(s) on alkali treatment. For further information see the text.

the main fraction of the resulting labelled polysaccharide chains emerged shortly after the excluded volume from a TSK G4000 SW column (Fig. 5a). Calibration of the column with hyaluronan standards of known $M_{\mathrm{r}}$ (results not shown) indicated an $M_{\mathrm{r}}$ of approx. 110000 (corresponding to about 280 disaccharide units) for this fraction. On the other hand, the chains produced at high UDP-GlcNAc concentration $(2.5 \mathrm{~mm}$ or $5 \mathrm{~mm})$ emerged appreciably retarded on the same column (Fig. $5 b$ ), as two broad poorly separated peaks corresponding to $M_{\mathrm{r}}$ approx. 45000 (about 110 disaccharide units) and less than 6000 (about 15 disaccharide units) respectively. These findings corroborate the relationship between rate of polymerization and polysaccharide chain length. Polysaccharide synthesized at an apparently intermediary rate (in the absence of exogenous UDP-GlcNAc; see Fig. 2) showed an elution pattern (Fig. 5c) intermediate between those of polysaccharides synthesized at high (Fig. $5 a$ ) or at low (Fig. $5 b$ ) rate.

Fig. 6 illustrates the effects of incubation time on polysaccharide chain length. The low rate of monomer 


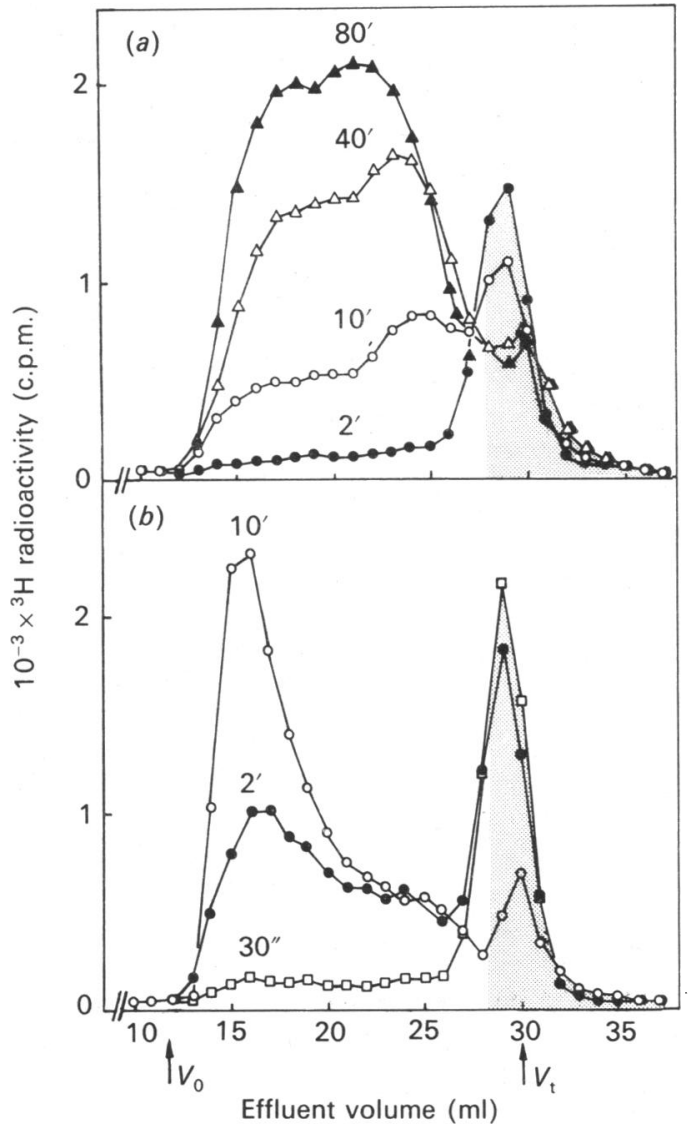

Fig. 6. Gel chromatography of polysaccharide chains synthesized for different periods of time at (a) high or (b) low UDPGlcNAc concentration

Mastocytoma microsomal fraction was incubated under standard conditions at $37^{\circ} \mathrm{C}$ (final volume $300 \mu \mathrm{l}$ ) with UDP- $\left[{ }^{3} \mathrm{H}\right] \mathrm{GlcA}$ and either (a) $5 \mathrm{~mm}$ - or (b) $25 \mu \mathrm{M}$-UDPGlcNAc for periods of time ranging from $30 \mathrm{~s}\left(30^{\prime \prime}\right)$ to $80 \mathrm{~min}\left(80^{\prime}\right)$ as indicated. Labelled polysaccharide was isolated and treated with alkali, and the resulting free polysaccharide chains were subjected to chromatography on a column of Ultropac TSK G4000 SW (see the Experimental section). The amounts of labelled polysaccharides in the chromatograms do not indicate the yields of the various incubations. For explanation of the stippled peaks see the legend to Fig. 5.

incorporation observed at high UDP-GlcNAc and low UDP-GlcA concentrations was reflected by slow chain elongation, as shown by gel chromatography of samples isolated after different periods of incubation (Fig. 6a). Chains isolated after $10 \mathrm{~min}$ showed a broad size distribution and tended to accumulate in two poorly resolved peaks. During prolonged incubation this pattern was slowly shifted towards lower elution volume, yet with the major peak at a $K_{\mathrm{av}}$ value of approx. $0.5\left(M_{\mathrm{r}}\right.$ approx. 20000) still after $80 \mathrm{~min}$ of incubation. In contrast, the chains formed in the presence of $25 \mu \mathrm{M}$ UDP-GlcNAc were large, with a peak $K_{\mathrm{av}}$ of approx. $0.25\left(M_{\mathrm{r}}\right.$ approx. 75000$)$, after only 2 min of incubation (Fig. 6b).

An attempt was made to determine quantitatively the rate of chain elongation obtained in the presence of high concentrations of both UDP-GlcNAc and UDP-GlcA.

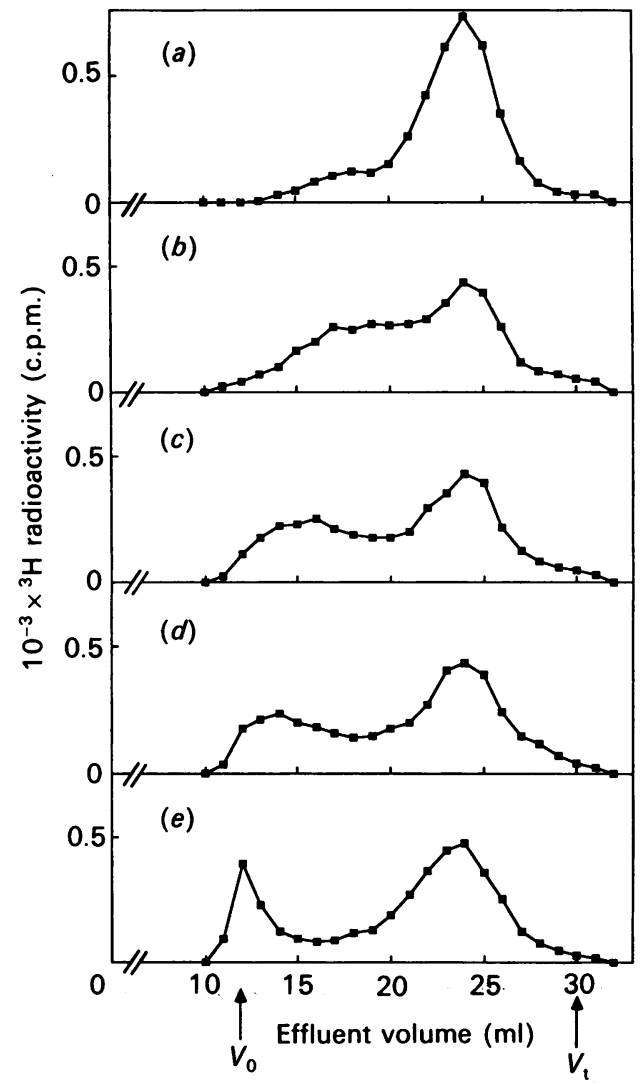

Fig. 7. Polysaccharide chain elongation at high concentrations of both UDP-GlcA and UDP-GlcNAc

Microsomal fraction was incubated at $37^{\circ} \mathrm{C}$ with UDP$\left[{ }^{3} \mathrm{H}\right] \mathrm{GlcA}$ and $5 \mathrm{~mm}$-UDP-GlcNAc. After $5 \mathrm{~min}$ of incubation, unlabelled UDP-GlcA was added to $5 \mathrm{~mm}$ concentration, and $300 \mu \mathrm{l}$ samples were withdrawn after (a) $0 \mathrm{~min},(b) 1 \mathrm{~min},(c) 5 \mathrm{~min},(d) 10 \mathrm{~min}$ and (e) $30 \mathrm{~min}$ of continued incubation. Each sample, mixed with an equal volume of $4 \% \mathrm{SDS}$, was treated at $100^{\circ} \mathrm{C}$ for $3 \mathrm{~min}$, and microsomal polysaccharide was isolated, treated with alkali, dialysed (to eliminate non-specifically incorporated label; see the legend to Fig. 4) and analysed by chromatography on Ultropac TSK G4000 SW as described in the Experimental section.

A pulse-chase approach was utilized, as illustrated in Fig. 7. Chains were labelled for $5 \mathrm{~min}$ at a low rate in the presence of UDP- $\left[{ }^{3} \mathrm{H}\right] \mathrm{GlcA}$ and $5 \mathrm{~mm}-\mathrm{UDP}-$ GlcNAc. The resulting retarded elution pattern was only slightly altered when the incubation time was extended by an additional $10 \mathrm{~min}$ period (results not shown). However, addition of UDP-GlcA at high concentration (5 mM), following the initial $5 \mathrm{~min}$ of pulse-labelling, resulted in a marked change in the elution profile of the pulse-labelled material (Figs. 7b-7e). After 1 min of chase incubation a major portion of less-retarded label was already observed (Fig. $7 b$ ), which was then progressively shifted towards the elution positions of larger molecules. Interestingly, only a fraction, varying between $30 \%$ and $60 \%$ in different experiments, of the pulse-labelled polysaccharide chains appeared to be amenable to elongation during the chase. The significance of this finding is unclear. The change in elution position of the major nascent chains would correspond to an increase in $M_{\mathrm{r}}$ of 150000-200000 during a $10 \mathrm{~min}$ period of chase 
incubation. Assuming an $M_{\mathrm{r}}$ for the repeating glucuronosyl- $N$-acetylglucosaminyl disaccharide unit of approx. 400, these results can be roughly approximated to indicate a rate of chain elongation during the chase of $30-40$ disaccharide units $/ \mathrm{min}$. The fraction of the chased polysaccharide that remained small-molecular could not be eliminated by further prolonging the chase period (results not shown).

\section{DISCUSSION}

Addition of UDP-GlcNAc to reaction mixtures containing mastocytoma microsomal fraction resulted in an increase in the rate of incorporation of label from UDP$\left[{ }^{3} \mathrm{H}\right] \mathrm{GlcA}$ into heparin-precursor polysaccharide. However, this effect applied only up to a point where the concentrations of the two UDP-sugars were approximately equal; a further increase in the UDP-GlcNAc concentration caused a decrease in the polymerization rate. A corresponding effect was observed when UDP$\left[{ }^{14} \mathrm{C}\right] \mathrm{GlcNAc}$ was added as the labelled precursor, together with unlabelled UDP-GlcA at different concentrations (results not shown). The inhibitory effect of either UDP-sugar on the polymerization reaction was noted only when the alternate precursor was present at relatively low concentration. Apparently, the inhibitory effect is not caused by the high UDP-sugar concentration as such but rather reflects a disproportion in the concentrations of the two sugar nucleotides and hence, presumably, a competition phenomenon.

Competition might be exerted at the glycosyltransferase level, UDP-GlcNAc at high concentration interacting with a nucleotide-binding site in the glucuronosyltransferase and thus interfering with the binding of the labelled UDP-GlcA to this enzyme; the reverse situation would apply to the $N$-acetylglucosaminyltransferase. On the other hand, if the catalytic sites of the glycosyltransferases are located within the vesicles of the Golgi system (Nuwayhid et al., 1986), the nucleotide sugars will have to be translocated across these membranes before they can interact with the enzymes. Capasso \& Hirschberg $(1984 a, b)$ have shown that the membranes contain specific transporters which mediate the equimolar exchange of cytosolic sugar nucleotides and 3'-phosphoadenylyl sulphate with the corresponding luminal nucleoside monophosphates. The nucleotide components, but not the sugar moieties, provide the recognition features prerequisite for binding to the Golgi membranes, but are not sufficient for overall translocation, which also depends on the type of sugar. The role of sugar nucleotide transport in the mastocytoma microsomal preparation used in the present study has not been evaluated. However, assuming the involvement of transporters for UDP-GlcNAc and UDP-GlcA that both bind UDP, the inhibition of polysaccharide chain formation observed at grossly disparate concentrations of the two sugar nucleotides may be explained in terms of mutual competition for binding to the appropriate transporter. At high concentration UDP-GlcNAc will not only saturate the UDP-GlcNAc transporter (resulting in a high rate of translocation of this sugar nucleotide) but will also compete with UDP-GlcA for binding to its transporter. If the latter component is present at low concentration it thus will be unable to penetrate the membrane, resulting in a lack of UDP-GlcA at the site of the polymerization reaction. Conversely, at high con- centration of UDP-GlcA and low concentration of UDP-GlcNAc translocation of the latter compound will be prevented. In accord with this interpretation, uridinebased nucleotides (but not uridine alone; see Table 1) were also able to inhibit polysaccharide synthesis, whereas adenosine-based nucleotides were less effective (cf. Capasso \& Hirschberg, 1984b). Again, regulation of glycosyltransferase activity through direct inhibition by intra-Golgi UDP or UMP remains a possibility.

Two principally different hypotheses have been advanced to define the factors that determine polysaccharide chain length in proteoglycan biosynthesis. One proposal implies that a specific structural modification of non-reducing terminal sugar unit, such as the incorporation of sulphate groups at certain positions, might serve as a chain-termination signal (Telser et al., 1966; Otsu et al., 1985). Effects of sulphation were not considered in the present study and thus cannot be evaluated. Alternatively, the length of polysaccharide chains is kinetically determined by the relation between polymerization activity and the number of available primer sites (Mitchell \& Hardingham, 1982). In accord with the latter hypothesis, the length of polysaccharide chains formed in our cell-free biosynthetic system could be modulated in a systematic fashion by varying the concentrations of the UDP-sugar precursors and thus the rate of the polymerization reaction.

Unfortunately, the mode of primer utilization remains obscure. The microsomal fraction obviously contains an appreciable pool of preformed primer sites for the major chain-elongation reaction, i.e. the formation of GlcAGlcNAc disaccharide units. Although chondroitin sulphate chains, apparently capable of further chain elongation, could be demonstrated in the microsomal preparation, the experiments did not reveal any heparinrelated polysaccharide. We therefore suggest that heparin-precursor chains were synthesized de novo in the cell-free system on primers containing the galactosylgalactosyl-xylosyl trisaccharide sequence of the polysaccharide-protein linkage region (such components would presumably have escaped detection by the analyses performed) but little or no additional saccharide. On the other hand, the experimental data imply that the primer pool is functionally heterogeneous, such that different sets of primer molecules are utilized under different conditions of chain elongation. Incubation of the microsomal fraction with UDP- $\left[{ }^{3} \mathrm{H}\right] \mathrm{GlcA}$ thus yielded the same amounts of labelled polysaccharide after $20 \mathrm{~min}$ in the presence of $25 \mu \mathrm{M}$-UDP-GlcNAc as after $40 \mathrm{~min}$ in the presence of $5 \mathrm{~mm}$-UDP-GlcNAc (Fig. 1); yet the polysaccharide chains isolated after such incubations differed drastically in size, being much larger after synthesis at the lower UDP-GlcNAc concentration (Fig. 6). This finding indicates that fewer chains were formed and hence that a smaller proportion of primer sites was utilized under the latter conditions. Could it be that different primer pools give rise to separate subpopulations of proteoglycans, synthesized with different kinetics? Indeed, such a possibility would be consistent with the marked polydispersity of the liberated polysaccharide chains, maintained also after prolonged incubation, as well as with the accumulation of these chains into discernible, albeit poorly resolved, subfractions (Figs. 5 and 6). Similar considerations apply to the experiment illustrated in Fig. .7, where only a fraction of the polysaccharide chains initiated during $5 \mathrm{~min}$ of 
pulse-labelling remained accessible for chase elongation. Did all pulse-labelled chains originate from a single pool of primer? A better characterization of the structure, distribution and commitment of primer saccharides will obviously promote our understanding of the overall process of proteoglycan biosynthesis.

We are grateful to $\mathrm{R}$. Cleland and $\mathrm{O}$. Wik for gifts of hyaluronan fractions of defined $M_{\mathrm{r}}$. This work was supported by Grant 2309 from the Swedish Medical Research Council and Grant 600/83D98:2 from the Swedish Council for Forestry and Agricultural Research, by the National Swedish Board for Technical Development, by KabiVitrum AB, Stockholm, Sweden, and by the Faculty of Veterinary Medicine, Swedish University of Agricultural Sciences. This is Paper XV of a series in which the preceding reports are by Jacobsson et al. (1985) and Riesenfeld et al. (1987).

\section{REFERENCES}

Balazs, E. A., Laurent, T. C. L. \& Jeanloz, R. W. (1986) Biochem. J. 235, 903

Bitter, T. \& Muir, H. M. (1962) Anal. Biochem. 4, 330-334

Capasso, M. J. \& Hirschberg, C. B. (1984a) Proc. Natl. Acad. Sci. U.S.A. 81, 7051-7055

Capasso, M. J. \& Hirschberg, C. B. (1984b) Biochim. Biophys. Acta 777, 133-139

Cleland, R. L. (1983) Biopolymers 22, 2501-2506

Furth, J., Hagen, P. \& Hirsch, E. I. (1957) Proc. Soc. Exp. Biol. Med. 95, 824-828

Gallagher, J. T., Lyon, M. \& Steward, W. T. (1986) Biochem. J. 236, 313-325

Höök, M., Lindahl, U., Hallén, A. \& Bäckström, G. (1975) J. Biol. Chem. 250, 6065-6071

Received 30 November 1987/16 February 1988; accepted 24 May 1988
Jacobsson, I. \& Lindahl, U. (1980) J. Biol. Chem. 255, 5094-5100

Jacobsson, K.-G. \& Lindahl, U. (1987) Biochem. J. 246, 409-415

Jacobsson, I., Bäckström, G., Höök, M., Lindahl, U., Feingold, D. S., Malmström, A. \& Rodén, K. (1979a) J. Biol. Chem. 254, 2975-2982

Jacobsson, I., Höök, M., Pettersson, I., Lindahl, U., Larm, O., Wirén, E. \& von Figura, K. (1979b) Biochem. J. 179, 77-87

Jacobsson, K.-G., Riesenfeld, J. \& Lindahl, U. (1985) J. Biol. Chem. 260, 12154-12159

Lindahl, U. \& Kjellén, L. (1986) in The Biology of the Extracellular Matrix: Biology of Proteoglycans (Wight, T. N. \& Mecham, R. P., eds.), pp. 59-104, Academic Press, New York

Lindahl, U., Bäckström, G., Jansson, L. \& Hallén, A. (1973) J. Biol. Chem. 248, 7234-7241

Mitchell, D. \& Hardingham, T. (1982) Biochem. J. 202, 387-395

Nuwayhid, N., Glaser, J. H., Johnson, J. C., Conrad, H. E., Hauser, S. C. \& Hirschberg, C. B. (1986) J. Biol. Chem. 261, 12936-12941

Otsu, K., Hirofumi, I., Tsuzuki, Y., Yonekura, H., Nakanishi, Y. \& Suzuki, S. (1985) Biochem. J. 227, 37-48

Poole, A. R. (1986) Biochem. J. 236, 1-14

Riesenfeld, J., Pettersson, I., Lindahl, U., Vann, W. F. \& Rodén, L. (1987) Glycoconjugate J. 4, 179-189

Robinson, H. C., Horner, A. F., Höök, M., Ögren, S. \& Lindahl, U. (1978) J. Biol. Chem. 253, 6687-6693

Rodén, L. (1980) in The Biochemistry of Glycoproteins and Proteoglycans (Lennarz, W. J., ed.), pp. 267-371, Plenum Publishing Corp., New York

Silbert, J. E. (1963) J. Biol. Chem. 238, 3542-3546

Telser, A., Robinson, H. C. \& Dorfman, A. (1966) Arch. Biochem. Biophys. 116, 458-465 